\title{
YOUNG MODULES FOR SYMMETRIC GROUPS
}

\author{
KARIN ERDMANN
}

\author{
To Laci Kovács on his 65 th birthday
}

(Received 1 February 2001; revised 15 May 2001)

Communicated by R. A. Bryce

\begin{abstract}
Let $K$ be a field of characteristic $p$. The permutation modules associated to partitions of $n$, usually denoted as $M^{\lambda}$, play a central role not only for symmetric groups but also for general linear groups, via Schur algebras. The indecomposable direct summands of these $M^{\lambda}$ were parametrized by James; they are now known as Young modules; and Klyachko and Grabmeier developed a 'Green correspondence' for Young modules. The original parametrization used Schur algebras; and James remarked that he did not know a proof using only the representation theory of symmetric groups. We will give such proof, and we will at the same time also prove the correspondence result, by using only the Brauer construction, which is valid for arbitrary finite groups.
\end{abstract}

2000 Mathematics subject classification: primary $20 \mathrm{C} 30,20 \mathrm{C} 20$.

\section{1. $p$-permutation modules via the Brauer construction}

1.1. Let $G$ be a finite group; we consider finite-dimensional $K G$-modules. We follow the approach of Broué from [1]. A module $M$ is said to be a ' $p$-permutation module' if for any $p$-subgroup $P$ of $G$ there is a $P$-invariant basis of $M$. The indecomposable $p$-permutation modules are, up to isomorphism, precisely the indecomposable summands of transitive permutation modules (or the trivial source modules).

In [1], a main result is the parametrization of indecomposable $p$-permutation modules by using the Brauer construction. This is defined as follows. Let $P$ be a

(C) 2001 Australian Mathematical Society 0263-6115/2001 \$A2.00+0.00 
(non-trivial) $p$-subgroup of $G$, and let $\bar{N}:=N_{G}(P) / P$. Then we have a functor

$$
-(P): \bmod -K G \rightarrow \bmod -K \bar{N}
$$

which takes a $K G$-module $V$ to $V(P)$, where

$$
V(P):=V^{P} / \sum_{Q<P} \operatorname{Tr}_{Q}^{P}\left(V^{Q}\right)
$$

Here $V^{R}$ are the fixed points under the action of $R$, and $\operatorname{Tr}_{Q}^{P}$ is the linear map from $V^{Q}$ to $V^{P}$ defined by $\operatorname{Tr}_{Q}^{P}(v)=\sum_{i} g_{i} v$, the sum taken over some transversal for the cosets of $Q$ in $P$.

Now suppose that $V$ is a $p$-permutation module. Take a $P$-stable basis $\mathscr{B}$ of $V$, then $\mathscr{B}$ is a union of $P$-orbits and $V^{P}$ is spanned by orbit sums, and an orbit sum is of the form $\operatorname{Tr}_{Q}^{P}(b)$ where $Q$ is the stabilizer of $b$, with $b \in \mathscr{B}$. To get $V(P)$ we require $Q=P$, and we identify $V(P)$ with the $K$-span of the fixed points of $P$ in $\mathscr{B}$. We will apply the following, which was suggested by Puig and published in [1].

CORRESPONDENCE THEOREM ([1]). Let $P$ be a $p$-subgroup of $G$. The functor $-(P)$ induces a 1-1 correspondence between the isomorphism classes of

(i) indecomposable p-permutation $K G$-modules with vertex $P$, and

(ii) indecomposable projective $K \bar{N}$-modules.

In particular, if $M$ is any $p$-permutation module and we have a direct sum decomposition $M=\oplus Y_{i}$ with all $Y_{i}$ indecomposable, then $Y_{i}$ has vertex $P$ if and only if $Y_{i}(P)$ is indecomposable projective as a module for $\bar{N}$. Suppose $Y$ is any indecomposable $p$-permutation module with vertex $P$, then $Y \cong Y_{i}$ if and only if $Y_{j}(P) \cong Y_{i}(P)$. We remark that $Y(P)$ (as a module for $N_{G}(P)$ ) is isomorphic to the Green correspondent in $N_{G}(P)$ of $Y$.

1.2. In our context, we have a $p$-permutation module $M$ and we have to identify the vertices of its indecomposable summands. It happens sometimes that $M(P)$ is zero for all non-trivial $p$-subgroups of $G$; then by the correspondence theorem, $M$ is projective. Moreover, not all $p$-groups are possible as vertices.

LEMMA 1. Suppose $M$ is any p-permutation module. If $Q$ is a $p$-subgroup of $G$ and $P$ is a proper subgroup of $Q$ such that $M(P)=M(Q)$, then $M$ does not have an indecomposable summand with vertex $P$.

PrOOF. Suppose $M(P)=M(Q)$, and we may assume that this is non-zero. Since $P$ is a proper subgroup of $Q$ there is some subgroup $P<R \leq Q$ such that $P$ is normal in $R$. Then $R / P$ acts trivially on $M(P)$, on the other hand a non-zero projective 
module for $N_{G}(P) / P$ cannot have a summand on which a normal $p$-subgroup acts trivially (the restriction to such subgroup must be projective and hence free). Hence $M(P)$ does not have a summand which is projective as a module for $N_{G}(P) / P$. By the Correspondence Theorem, $M$ does not have a summand with vertex $P$.

\section{Young modules}

2.1. From now we assume that $G$ is the symmetric group $\mathscr{S}_{r}$ of degree $r$. For each partition $\lambda$ of $r$ there is a transitive permutation module $M^{\lambda}$, on the cosets of any 'Young subgroup' of type $\lambda$, that is, a direct product of symmetric groups of degrees $\lambda_{1}, \lambda_{2}, \ldots, \lambda_{k}$ acting on disjoint subsets of $\{1,2, \ldots, r\}$, where $\lambda_{1}, \ldots, \lambda_{k}$ are the non-zero parts of $\lambda$. We denote any such subgroup by $\mathscr{S}_{\lambda}$ (it will not be necessary to specify it further). Moreover, we will call such $M^{\lambda}$ a 'Young permutation module'. Actually, $\lambda$ is allowed to be an unordered partition.

Following [3,5], we will take for the underlying $G$-set the row equivalence classes of $\lambda$-tableaux. Recall that a $\lambda$-tableau is an array of shape $\lambda$ where the entries are filled with the numbers from 1 to $r$, allowing no repeats. The group $G$ acts naturally (on the right) on the set of all $\lambda$-tableaux. Fix a $\lambda$-tableaux $t$, then its row stabilizer $R(t)$ is the subgroup of $G$ keeping the rows fixed as a set, and similarly one defines $C(t)$, the column stabilizer. Suppose $t_{1}$ and $t_{2}$ are $\lambda$-tableaux, then row equivalence $\sim$ is defined as

$$
t_{1} \sim t_{2} \quad \Longleftrightarrow \quad t_{2}=t_{1} \pi \quad \text { (some } \pi \in R(t) \text { ). }
$$

Write $\{t\}$ for the equivalence class of $t$ under row equivalence, and call $\{t\}$ a $\lambda$-tabloid. The group $G$ acts on the set of $\lambda$-tabloids, where the action is induced by the natural action; this is clearly transitive, and $M^{\lambda}$ is the corresponding permutation module, we take as basis the set $\mathscr{B}$ of all $\lambda$-tabloids $\{t\}$. Write $\leq$ for the dominance order of partitions (see [3, 5]).

The main results in [4], whose statements involve only modules of symmetric groups, are as follows.

THEOREM 1. There is a set of indecomposable $K \mathscr{S}_{r}$-modules $Y^{\mu}$ one for each partition $\mu$ of $r$, such that the following hold for all partitions $\lambda$ of $r$ :

(i) $M^{\lambda}$ is a direct sum of $K \mathscr{S}_{r}$-modules, each of which is isomorphic to some $Y^{\mu}$ with $\mu \geq \lambda$, and precisely one summand is isomorphic to $Y^{\lambda}$.

(ii) If $Y^{\lambda} \cong Y^{\mu}$, then $\lambda=\mu$.

2.2. The definition of the Young modules involves properties of Specht modules. Let $t$ be any $\lambda$-tableau, and let $\mathscr{V}^{t} \in K G$ be the alternating sum of the column stabilizer 
$C(t)$, and define

$$
e_{t}=\{t\} \mathscr{V}^{t}
$$

an element in $M^{\lambda}$. The Specht module $S^{\lambda}$ is defined to be the $K G$-submodule of $M^{\lambda}$ generated by $e_{t}$. It was proved by James that

$$
\left(M^{\lambda}\right) \mathscr{V}^{t}=K e_{t} \text {, and if } \beta \geq \lambda \text { then }\left(M^{\beta}\right) \mathscr{V}^{t}=0 .
$$

This is proved using symmetric groups only (see $[5,7.1 .5])$. On $M^{\lambda}$, we have a bilinear form with respect to which the above basis is orthonormal. This is non-degenerate and also $\mathscr{S}_{r}$-invariant. James' Submodule Theorem follows now from the first part of $(*)$ :

If $A$ is a submodule of $M^{\lambda}$, then either $S^{\lambda} \subseteq A$ or $A \subseteq\left(S^{\lambda}\right)^{\perp}$.

Write $M^{\lambda}$ as a direct sum of indecomposable $K \mathscr{S}_{r}$-modules. Then by the Submodule Theorem, there is a unique indecomposable summand which contains $S^{\lambda}$, and we denote this by $Y^{\lambda}$, and call it the Young module associated to $\lambda$. In fact, we can even say that if $Y$ is an indecomposable summand of $M^{\lambda}$ such that $Y \cap S^{\lambda} \neq 0$, then $Y \cong Y^{\lambda}$.

The second part of $(*)$ implies part of James' Theorem; namely $Y^{\lambda}$ is a direct summand of $M^{\beta}$, then $\left(M^{\beta}\right) \mathscr{V}^{t} \supseteq\left(S^{\lambda}\right) \mathscr{Y}^{t} \neq 0$ and hence $\beta \geq \lambda$. Furthermore, a similar argument shows that $Y^{\lambda} \cong Y^{\mu}$ only if $\lambda=\mu$. These are the arguments from [4]. Our aim is to prove part (i) of Theorem 1, by using the correspondence of $p$-permutation modules given in Broué's theorem. This naturally proves Grabmeier's result at the same time.

2.3. In [2], Grabmeier shows that each Young module $Y^{\lambda}$ has a 'Young vertex'. This is a minimal Young subgroup $\mathscr{S}_{\rho}$, unique up to conjugation, such that $Y$ is a direct summand of $M^{\rho}$; and $\rho$ is a partition with parts only $p$-powers $\geq 1$. Then he proves that there is a 1-1 correspondence between the indecomposable summands of the Young permutation modules with Young vertex $\mathscr{S}_{\rho}$ and the indecomposable projective modules of $N_{G}\left(\mathscr{S}_{\rho}\right) / \mathscr{S}_{\rho}$ (see $[2,7.8]$ ). The correspondent (for $Y^{\lambda}$ nonprojective) is basically the Green correspondent with respect to the $N_{G}\left(\mathscr{S}_{p}\right)$, on which the group $\mathscr{S}_{\rho}$ acts trivially and which can therefore be viewed as a module for the factor group. Actually, Grabmeier does not explicitly state that the $G$-module of the correspondence is what we call the Young module; but it would follow if one would work out the multiplicities in Klyachko's formula (see [2, 7.14]). We remark that Grabmeier's proof of the formula uses symmetric groups only (whereas Klyachko's approach is via the Schur algebra). The correspondence is as follows. 
THEOREM 2. The Young module $Y^{\lambda}$ is projective if and only if $\lambda$ is $p$-restricted.

Suppose $\lambda$ is not restricted, and write $\lambda=\sum_{m=0}^{s} \lambda(m) p^{m}$ with $\lambda(m) p$-restricted, let $r_{m}$ be the degree of $\lambda(m)$. Let $\rho$ be the partition of $r$ which has $r_{m}$ parts equal to $p^{m}$. Then

(i) $\mathscr{S}_{\rho}$ is a Young vertex of $Y^{\lambda}$, and

(ii) The Green correspondent of $Y^{\lambda}$ in $N_{G}\left(\mathscr{S}_{\rho}\right)$ is $Y^{\lambda(0)} \otimes Y^{\lambda(1)} \otimes \cdots \otimes Y^{\lambda(s)}$, as a module for the quotient $N_{G}\left(\mathscr{S}_{\rho}\right) /\left(\mathscr{S}_{\rho}\right)$.

A partition $\lambda=\left(\lambda_{1}, \lambda_{2}, \ldots, \lambda_{k}\right)$ is $p$-restricted if $\lambda_{i}-\lambda_{i+1}<p$ for $1 \leq i \leq k-1$ and also $\lambda_{k}<p-1$. We will refer to such expansion of $\lambda$ with $\lambda(m)$ restricted (which exists and is unique) as 'the $p$-adic expansion'.

\section{Identifying $M^{\lambda}(P)$}

3.1. Let $P$ be any non-trivial $p$-subgroup of $\mathscr{S}_{r}$, and let $\lambda$ be a partition of $r$. Then a basis element $\{t\}$ of $M^{\lambda}$ is fixed by $P$ if and only if the rows of $t$ are unions of $P$-orbits. If $P<Q$ and $Q$ has the same orbits as $P$, then $M^{\lambda}(P)=M^{\lambda}(Q)$. So Lemma 1 shows that the possible vertices of summands of $M^{\lambda}$ are Sylow $p$-subgroups of Young subgroups $\mathscr{S}_{\rho}$ where $\rho$ is a partitions whose parts are powers of $p$.

Let $\rho$ be a partition of $r$ which has $r_{i}$ parts equal to $p^{i}$, where $\sum_{m=1}^{s} r_{i} p^{i}=r$, let $\mathscr{S}_{\rho}$ be a corresponding Young subgroup, and moreover let $P$ be a Sylow $p$-subgroup of $\mathscr{S}_{\rho}$. The Frattini argument shows that the normalizer in $G$ of $\mathscr{S}_{\rho}$ is contained in $N_{G}(P) \mathscr{S}_{\rho}$; and since the normalizer of $\mathscr{S}_{\rho}$ is precisely the group of permutations which permutes the $P$ - (and $\mathscr{S}_{\rho}$ )-orbits amongst themselves, we have:

LEMMA 2. We have $N_{G}(P) \mathscr{S}_{\rho}=N_{G}\left(\mathscr{S}_{\rho}\right)$, and hence $N_{G}\left(\mathscr{S}_{\rho}\right) / \mathscr{S}_{\rho} \cong N_{G}(P) /$ $N_{\mathscr{S}_{\rho}}(P)$. This is isomorphic to the Young subgroup $\mathscr{S}_{r_{0}} \times \mathscr{S}_{r_{1}} \times \cdots \times \mathscr{S}_{r_{s}}$.

Returning to the permutation modules, we see directly that $\mathscr{S}_{p}$ acts trivially on $M^{\lambda}(P)$. So the structure as a module for $N_{G}(P) / P$ is the same as the structure as a module for $N_{G}(P) / N_{\mathscr{S}_{p}}(P)$, which by the lemma is isomorphic to the Young subgroup $\mathscr{S}_{\beta}$, where $\beta=\left(r_{0}, r_{1}, \ldots, r_{s}\right)$ is an unordered partition. Note that this also shows that the 'Grabmeier correspondence' is the same as the correspondence by the Brauer construction.

3.2. We will now view $M^{\lambda}(P)$ as a module for the Young subgroup $\mathscr{S}_{\beta}$, which acts on the $P$-orbits and where $\mathscr{S}_{r_{m}}$ permutes the orbits of length $p^{m}$ and fixes all other orbits. For each partition $\alpha(m)$ of $\mathscr{S}_{r_{m}}$ we have the Young permutation module $M^{\alpha(m)}$, and the outer tensor product $M^{\alpha(0)} \otimes M^{\alpha(1)} \otimes \cdots \otimes M^{\alpha(s)}$ is then a module for $\mathscr{S}_{\beta}$. The $\mathscr{S}_{\beta}$-module $M^{\lambda}(P)$ is isomorphic to a direct sum of such tensor procucts. More precisely 
PROPOSITION 1. As a module for $\mathscr{S}_{\beta}, M^{\lambda}(P)$ is isomorphic to the direct sum

$$
\bigoplus_{\tilde{\alpha}}\left(M^{\alpha(0)} \otimes M^{\alpha(1)} \otimes \cdots \otimes M^{\alpha(s)}\right),
$$

where the sum is taken over all $\tilde{\alpha}:=(\alpha(0), \alpha(1), \ldots, \alpha(s))$ such that

$$
\lambda=\sum_{m=1}^{s} \alpha(m) p^{m}
$$

and $\alpha(m)$ is an unordered partition of $r_{m}$.

ProOF. We fix a labelling of the $P$-orbits of length $p^{m}$ as $\Theta_{1}, \ldots, \Theta_{r_{m}}$, for each $m$. Take a $\lambda$-tableau $t$ such that $\{t\}$ is fixed by $P$, so that the rows of $t$ are unions of $P$-orbits. Define $\alpha(m)$ by setting $\alpha(m)_{i}:=$ the number of $P$-orbits of length $p^{m}$ in the $i$-th row of $t$. So we have $\lambda_{i}=\sum_{m} \alpha(m)_{i} p^{m}$ and $\lambda=\sum_{m} \alpha(m) p^{m}$; and $\alpha(m)$ is an unordered partition of $r_{m}$.

Now let $t_{m}$ be an $\alpha(m)$-tableau whose $i$-th row consists of all $j$ such that the $j$-th $P$-orbit of length $p^{m}$ belongs to the $i$-th row of $t$. Then set

$$
\psi(\{t\}):=\left\{t_{0}\right\} \otimes\left\{t_{1}\right\} \otimes \cdots \otimes\left\{t_{s}\right\} .
$$

This belongs to $M^{\alpha(0)} \otimes M^{\alpha(1)} \otimes \cdots \otimes M^{\alpha(s)}$. The linear map defined by $\psi$ gives a vector space isomorphism between $M^{\lambda}(P)$ and the direct sum of all such tensor products. It remains to show that it is also a homomorphism of $\bar{N}$-modules.

Let $g \in N_{G}(P)$ and let $\bar{g} \in \mathscr{S}_{\beta}$ be the corresponding permutations of the $P$-orbits. Then we have $\bar{g}=\Pi g_{i}$, where $g_{i} \in \mathscr{S}_{r_{i}}$, so that $g_{i}$ permutes the orbits of length $p^{i}$ and fixes every other orbit. Write $\{g t\}=\{\tilde{t}\}$, the $j$-th $P$-orbit $\Theta_{j}$ of length $p^{m}$ occurs in the $\mathrm{i}$-th row of $t$ if and only if $g \Theta_{j}$ occurs in the $i$-th row of $\tilde{t}$, that is if and only if $g_{m}(j)$ occurs in the i-th row of $\psi(\{\tilde{t}\})$. But these $g_{m}(j)$ form also the $i$-th row of $\bar{g} \psi(\{t\})$. This shows that $\psi(g\{t\})=\bar{g} \psi(\{t\})$.

3.3. Now let $\lambda$ be a partition of $r$ and suppose it has $p$-adic expansion

$$
\lambda=\lambda(0)+\lambda(1) p+\cdots+\lambda(s) p^{s} .
$$

Let $r_{m}$ be the degree of $\lambda(m)$ for $0 \leq m \leq s$, and let $\rho$ be the partition of $r$ which has $r_{m}$ parts equal to $p^{m}$. Take $P$ to be a Sylow subgroup of $\mathscr{S}_{\rho}$. Then by Section 3.2 we know that $M^{\lambda}(P)$ has a summand $M^{\tilde{\lambda}}$ which we define as

$$
M^{\bar{\lambda}}:=M^{\lambda(0)} \otimes M^{\lambda(1)} \otimes \cdots \otimes M^{\lambda(k)}
$$

We wish to identify the image of $Y^{\lambda}$ in $M^{\lambda}(P)$ as a specific summand of $M^{\tilde{\lambda}}$; and we will do this by considering the image of $S^{\lambda}$ in $M^{\lambda}(P)$. 
LEMMA 3. There is some $\lambda$-tableau such that $\{t\}$ is fixed by $P$, and such that the image of the Specht module generator $e_{t}$ in $M^{\lambda}(P)$ is identified with the element $e_{t_{0}} \otimes e_{t_{1}} \otimes \cdots \otimes e_{t_{k}}$, in $M^{\bar{\lambda}}$, where $e_{t_{i}}$ generates the Specht module $S^{\lambda(i)}$.

ProOF. Take any $t$ such that $\{t\}$ in $M^{\lambda}(P)$ corresponds to an element of $M^{\bar{\lambda}}$ under the map $\psi$. We want to find the image of $e_{t}$ in $M^{\lambda}(P)$. Let $D=C(t) \cap N_{G}(P) \mathscr{S}_{\rho}$, and let $\mathscr{V}(D)$ be the alternating sum of the elements of $D$. Then we can write

$$
\mathscr{V}^{t}=\sum_{i} s\left(\pi_{i}\right) \mathscr{V}(D) \pi_{i}
$$

where the sum is taken over a set of coset representatives; and we take $\pi_{1}=1$. (Here $s(\pi)$ denotes the sign of the permutation $\pi$.) Then a tabloid occurring in $\{t\} \mathscr{V}^{t}$ is fixed by $P$ if and only if it occurs in $\{t\} \mathscr{V}(D)$, that is, the image of $e_{t}$ in $M^{\lambda}(P)$ is $\{t\} \mathscr{V}(D)$. This corresponds under $\psi$ to $\psi(\{t\}) \mathscr{V}(D)$, which is equal to $\left(\left\{t_{0}\right\} \otimes \cdots \otimes\left\{t_{s}\right\}\right) \mathscr{V}(D)$.

The image of $D$ in $\mathscr{S}_{\beta}$ is contained in the product $C$, where $C:=C\left(t_{0}\right) \times C\left(t_{1}\right) \times$ $\cdots \times C\left(t_{s}\right)$. If we find $t$ such that both are equal then we are done.

We take a tableau $t$ which has $\lambda(m)_{i}$ orbits of size $p^{m}$ in row $i$, for each $m$ and for each $i$ (with $1 \leq i \leq k$ if $\lambda$ has $k$ non-zero parts). To ensure that every permutation in $C$ is in the image of $D$ we must find $t$ with the following properties. Whenever entries $x$ and $y$ are in the same column of $t$ then they must belong to $P$-orbits of the same length. Moreover, for any other point $z$ in the row of $x$ which lies in the same $P$-orbit as $x$, the entry in the same column as $z$ and in the same row as $y$ must be in the same $P$-orbit as $y$. This ensures that there is a permutation in $D$ which interchanges these two orbits, and its image in $C$ is a 2 -cycle in the relevant factor.

One constructs such $t$ as follows. Order the entries in the last row arbitrarily. Then consider the row above the last row. It has at least as many orbits of size $p^{m}$ as the last row (since $\lambda(m)$ is a partition). Above the points in the last row in an orbit of size $p^{m}$, arrange therefore the points in some orbit of size $p^{m}$. Do so for each $m$. Then arrange the remaining entries in the last but one row arbitrarily. Repeat this procedure, working upwards.

3.4. We need a generalization of James' Submodule Theorem. Let $\mathscr{S}_{\beta}:=\mathscr{S}_{r_{0}} \times$ $\mathscr{S}_{r_{1}} \times \cdots \times \mathscr{S}_{r_{s}}$ be a direct product of symmetric groups, acting on disjoint sets, and let $\alpha(m)$ be a partition of $r_{m}$, for $0 \leq m \leq s$. Let

$$
M^{\tilde{\alpha}}:=M^{\alpha(0)} \otimes M^{\alpha(1)} \otimes \cdots \otimes M^{\alpha(s)}
$$

the (outer) tensor product of permutation modules; this is a module for the Young subgroup $\mathscr{S}_{\beta}$. This has basis consisting of all $\left\{t_{0}\right\} \otimes \cdots \otimes\left\{t_{s}\right\}$, where $\left\{t_{m}\right\}$ runs through the natural basis of $M^{\alpha(m)}$. Then this module $M^{\tilde{\alpha}}$ contains the tensor product of the 
Specht modules $S^{\tilde{\alpha}}:=S^{\alpha(0)} \otimes \cdots \otimes S^{\alpha(s)}$, that is, the module generated by

$$
e_{i}:=e_{t_{0}} \otimes e_{t_{1}} \otimes \cdots \otimes e_{t_{s}} .
$$

Then $Y^{\bar{\alpha}}:=Y^{\alpha(0)} \otimes \cdots \otimes Y^{\alpha(s)}$ is an indecomposable summand of $M^{\bar{\alpha}}$ which contains $S^{\tilde{\alpha}}$. (Recall that the outer tensor product of indecomposable modules is always indecomposable.)

LEMMA 4. Write $M^{\dot{\alpha}}$ as a direct sum of indecomposable $\mathscr{S}_{\beta}$-modules. Then there is a unique indecomposable summand which contains $S^{\bar{\alpha}}$ and this is isomorphic to $Y^{\tilde{\alpha}}$.

PROOF. This follows by precisely the same arguments as James' proof. Take the bilinear form $(-,-)$ on $M^{\bar{\alpha}}$ such that the natural basis is orthonormal, this bilinear form is invariant under the action of the Young subgroup $\mathscr{S}_{\beta}$. Then if $t_{m}$ is a $\alpha(m)$ tableau, for $0 \leq m \leq s$, we set

$$
\mathscr{V}^{i}:=\mathscr{V}^{t_{0}} \otimes \cdots \otimes \mathscr{V}^{t_{s}}
$$

Then $e_{i}=\left(\left\{t_{0}\right\} \otimes\left\{t_{1}\right\} \otimes \cdots \otimes\left\{t_{k}\right\}\right) \mathcal{V}^{i}$, and $\left(M^{\tilde{\alpha}}\right) \mathscr{V}^{i}=K e_{i}$, by the original result of James (see Section 2.2). The claim follows now as in Section 2.2.

\section{The proof of the theorems}

4.1. So far we have determined a direct sum decomposition of $M^{\lambda}(P)$. Moreover, if $\lambda$ is not $p$-restricted then we have identified a direct summand of $M^{\lambda}(P)$ which is the candidate for $Y^{\lambda}(P)$ as given in Theorem 2. We also know that if a Young module $Y^{\mu}$ is a direct summand of $M^{\lambda}$ then $\mu \geq \lambda$, and moreover Young modules labelled by different partitions are not isomorphic (see Section 2.2). Note also that the number of $p$-restricted partitions of $r$ is the same as the number of indecomposable projective $K \mathscr{S}_{r}$-modules, up to isomorphism (since the simple modules can be labelled by $p$-restricted partitions).

In order to complete the proofs we need to show

(i) Every indecomposable summand of $M^{\mu}$ is a Young module.

(ii) A Young module $Y^{\lambda}$ is projective if and only if $\lambda$ is $p$-restricted.

(iii) Suppose $\lambda$ is not $p$-restricted, with $p$-adic expansion $\lambda=\sum \lambda(m) p^{m}$. Then with the notation as in Theorem 2 (ii), a vertex of $Y^{\lambda}$ is a Sylow $p$-subgroup of $\mathscr{S}_{\rho}$, and $Y^{\lambda}(P)=Y^{\lambda(0)} \otimes Y^{\lambda(1)} \otimes \cdots \otimes Y^{\lambda(s)}$.

4.2. We will prove these by induction on $r$.

(1) Assume first that $r<p$, then the group algebra $K \mathscr{S}_{r}$ is semi-simple. Each $Y^{\lambda}$ is therefore indecomposable projective, and each partition is $p$-restricted. The 
total number of Young modules is the same as the number of indecomposable projectives, hence every indecomposable projective is a Young module. Every module is projective, hence $M^{\mu}$ is a direct sum of Young modules.

(2) We consider $r=p$ briefly (although this could be left out). The only nontrivial vertex is a group $P$ generated by a $p$-cycle. We have $M^{(p)}=Y^{(p)}=K$ and $M^{(p)}(P)=K$; and $M^{\lambda}(P)=0$ otherwise. Hence $Y^{(p)}$ is the only non-projective Young module in this case; it has vertex $P$ and its correspondent is $Y^{(1)}$ as a module for $\mathscr{S}_{1}$, which proves (iii). Moreover $(p)$ is the only non-restricted partition and we have (ii). There are now as many projective Young modules as there are restricted partitions, so every projective is a Young module and hence (i) holds in this case.

(3) Now let $r>p$ and assume that (i) to (iii) hold for all degrees less than $r$. Consider first a partition $\lambda$ which is not $p$-restricted. Let $\lambda(m)$ and $\rho$ be as in (iii). Let $P$ be a Sylow $p$-subgroup of $\mathscr{S}_{\rho}$. By Section 3.2 we know that $M^{\lambda}(P)$ has a direct summand $M^{\tilde{\lambda}}:=M^{\lambda(0)} \otimes M^{\lambda(1)} \otimes \cdots M^{\lambda(s)}$ as a module for $\mathscr{S}_{\beta}=N_{G}\left(\mathscr{S}_{\rho}\right) / \mathscr{S}_{\rho}$, where $\beta=\left(r_{0}, \ldots, r_{s}\right)$. Moreover, by Section 3.3 there is some generator $e_{t}$ of the Specht module $S^{\lambda} \subseteq Y^{\lambda}$ such that the image of $e_{t}$ is non-zero in $S^{\bar{\lambda}}:=S^{\lambda(0} \otimes S^{\lambda(1)} \otimes \cdots \otimes S^{\lambda(s)}$, and $S^{\tilde{\lambda}}$ is contained in $Y^{\bar{\lambda}}$, which is a direct summand of $M^{\tilde{\lambda}}$.

By the inductive hypothesis, $Y^{\lambda(m)}$ is projective as $\mathscr{S}_{r_{m}}$-module and hence $Y^{\tilde{\lambda}}$ is projective as a module for $\mathscr{S}_{\beta}$. By the Correspondence Theorem, $M^{\lambda}$ has a summand with vertex $P$ corresponding to $Y^{\bar{\lambda}}$. We claim that this summand is $Y^{\lambda}$. By Section 3.3 we know that $S^{\lambda}(P) \cap Y^{\tilde{\lambda}}$ is non-zero and hence $Y^{\lambda}(P) \cap Y^{\tilde{\lambda}}$ is non-zero. Since $Y^{\bar{\lambda}}$ is projective (hence injective) it has a simple socle, which is also the socle of $S^{\bar{\lambda}}$, and we conclude that $Y^{\lambda}(P) \cap S^{\tilde{\lambda}}$ is non-zero. Now $Y^{\lambda}(P)$ is a summand of $M^{\lambda}(P)$, and by the uniqueness result of Section 3.4 we deduce that $Y^{\tilde{\lambda}}$ is a summand of $Y^{\lambda}(P)$. So $Y^{\lambda}$ has a summand with vertex $P$; but $Y^{\lambda}$ is indecomposable, and therefore $Y^{\lambda}$ has vertex $P$ and $Y^{\lambda}(P)=Y^{\tilde{\lambda}}$, and (iii) is proved.

We claim that we have already found all non-projective indecomposable summands of $M^{\lambda}$. Namely, take a non-trivial $p$-subgroup $P$ which could occur as a vertex of a non-projective summand, then $P$ is a Sylow $p$-subgroup of $\mathscr{S}_{\rho}$ where $\rho$ is a partition where all parts are $p$-powers. Say $\rho$ has $r_{m}$ parts equal to $p^{m}$. By Section 3.2, we have $M^{\lambda}(P)$ is the direct sum of modules $\left(M^{\alpha(0)} \otimes M^{\alpha(1)} \otimes \cdots \otimes M^{\alpha(s)}\right)$, where $\alpha(m)$ is an unordered partition of $r_{m}$ and $\lambda=\sum_{m} \alpha(m) p^{m}$. Let $\gamma(m)$ be the partition associated to $\alpha(m)$, then of course $M^{\alpha(i)} \cong M^{\gamma(i)}$. Since $P \neq 1$ we know that $\mathscr{S}_{r_{m}}$ has order less than $\left|\mathscr{S}_{r}\right|$. By the inductive hypothesis, $M^{\alpha(m)}$ is a direct sum of Young modules, and a summand is projective if and only if the labelling partition is restricted. Now, these have already been identified as correspondents of Young modules for $\mathscr{S}_{r}$.

Assume now $\lambda$ is restricted, the $Y^{\lambda}$ must be projective. The total number of these is the same as the number of indecomposable projectives and hence every projective is a Young module. We deduce again that (i) holds. This completes the proof of Theorem 1 and Theorem 2. 
4.3. The Correspondence Theorem shows that there are more $p$-permutation modules in general than just the Young modules. First, a vertex $P$ of a non-projective Young module must be a Sylow subgroup of the full permutation group, $\mathscr{S}_{\rho}$ say, with the same orbits. Second, the correspondents in $N_{G}(P) / P$ of Young modules with such vertex are precise those indecomposable projectives on which $\mathscr{S}_{\rho} \cap N_{G}(P) / P$ act trivially.

For example, consider $r=p$, and $P$ a Sylow subgroup of $\mathscr{S}_{p}$. Then we have only one Young module with vertex $P$ but there are $p-1 p$-permutation modules in total with vertex $P$, they correspond to the simple modules of $N_{G}(P) / P$. In this case it is not hard to identify the correspondents in $G$, they are the Specht modules $S^{\lambda}$, where $\lambda=\left(p-a, 1^{a}\right)$ and $a$ is even, $0 \leq 1 \leq p-1$, and the duals of these.

\section{References}

[1] M. Broué, 'On Scott modules and $p$-permutation modules: an approach through the Brauer morphism', Proc. Amer. Math. Soc. 93 (1985), 401-408.

[2] J. Grabmeier, 'Unzerlegbare Moduln mit trivialer Youngquelle und Darstellungstheorie der Schuralgebra', Bayreuth. Math. Schr. 20 (1985), 9-152.

[3] G. D. James, The representation theory of the symmetric group, Lecture Notes in Math. 682 (Springer, Berlin, 1978).

[4] __. 'Trivial source modules for symmetric groups', Arch. Math. 41 (1983), 294-300.

[5] G. D. James and A. Kerber, The representation theory of the symmetric group, Encyclopedia Math. 16 (Addison and Wesley, Reading, 1981).

[6] A. A. Klyachko, 'Direct summands of permutation modules', Selecta Math. Soviet. (1) 3 (1983/84), 45-55.

Mathematical Institute

24-29 St. Giles

Oxford OX1 3LB

United Kingdom

e-mail: erdmann@maths.ox.ac.uk 